\title{
Entropy and its variational principle for noncompact metric spaces
}

\author{
Mauro Patrão
}

October 26, 2018

\begin{abstract}
In the present paper, we introduce a natural extension of AKMtopological entropy for noncompact spaces and prove a variational principle which states that the topological entropy, the supremum of the measure theoretical entropies and the minimum of the metric theoretical entropies always coincide. We apply the variational principle to show that the topological entropy of automorphisms of simply connected nilpotent Lie groups always vanishes. This shows that the classical formula for the entropy of an automorphism of a noncompact Lie group is just an upper bound for its topological entropy.
\end{abstract}

AMS 2000 subject classification: Primary: 37B40 37A35, Secondary: 22E25. Key words: Topological entropy, variational principle, non-compact metric spaces, automorphisms of Lie groups.

\section{Introduction}

Topological entropy was introduced in [1] by Adler, Konheim and MacAndrew (AKM) to study the dynamic of a continuous map $T: X \rightarrow X$ defined on a compact space $X$. They have also conjectured the well known variational principle which states that

$$
h(T)=\sup _{\mu} h_{\mu}(T),
$$

where $h(T)$ is the topological entropy of $T, h_{\mu}(T)$ is the $\mu$-entropy of $T$ and the supremum is taken over all $T$-invariant probabilities $\mu$ on $X$. 
R. Bowen has extended in 2] the concept of topological entropy for noncompact metric spaces. This approach uses the concept of generator sets, which are defined by means of a given metric. For compact spaces this definition of entropy is, in fact, independent of the metric and, indeed, coincides with the AKM-topological entropy.

However, for noncompact spaces Bowen's entropy depends on the metric. In Lemma 1.5 of [4], it was proved the following variational principle for locally compact spaces

$$
\sup _{\mu} h_{\mu}(T)=\inf _{d} h_{d}(T),
$$

where $h_{d}(T)$ is the $d$-entropy of $T$ and the infimum is taken over all metric $d$ on $X$.

In the present paper, we improve this result showing that the above infimum is always attained at $d$, whenever $d$ is a metric satisfying some special conditions. Theses metrics are called admissible metrics and always exist when $X$ is a locally compact separable space. Moreover the supremum and the minimum coincide with a natural extension of AKM-topological entropy, introduced in the second section. In the third section, this variational principle is proven.

In the last section, we apply the variational principle to determine the topological entropy of automorphisms of some Lie groups. First we consider linear isomorphisms of a finite dimensional vector space and characterize their recurrent sets in terms of their multiplicative Jordan decompositions. We show that the topological entropy of these linear isomorphisms always vanishes. This shows that the classical formula for its $d$-entropy, where $d$ is the euclidian metric, is just an upper bound for its null topological entropy. Remember that this classical formula is given by

$$
h_{d}(T)=\sum_{|\lambda|>1} \log |\lambda|,
$$

where $\lambda$ runs through the eigenvalues of the linear isomorphism $T$ (cf. Theorem 8.14 in [7]). Thus it might be an interesting problem to calculate the topological entropy of a given automorphism $\phi$ of a general noncompact Lie group $G$, since the classical formula for its $d$-entropy, where $d$ is some invariant metric, is as well just an upper bound for its topological entropy. We conclude this paper providing an answer for this problem when $G$ is a simply connected nilpotent Lie group. We show that the topological entropy of its automorphisms also vanishes. 


\section{Topological entropy and admissible metrics}

We start this section presenting an extension of the AKM-topological entropy introduced in [1]. Let $X$ be a topological space and $T: X \rightarrow X$ be a proper map, i.e., $T$ is a continuous map such that the pre-image by $T$ of any compact set is compact. An admissible covering of $X$ is an open and finite covering $\alpha$ of $X$ such that, for each $A \in \alpha$, the closure or the complement of $A$ is compact. Given an admissible covering $\alpha$ of $X$, for every $n \in \mathbb{N}$, we have that the set given by

$$
\alpha^{n}=\left\{A_{0} \cap T^{-1}\left(A_{1}\right) \cap \ldots \cap T^{-n}\left(A_{n}\right): A_{i} \in \alpha\right\}
$$

is also an admissible covering of $X$, since $T$ is a proper map. Given an admissible covering $\alpha$ of $X$, we denote by $N\left(\alpha^{n}\right)$ the smallest cardinality of all sub-coverings of $\alpha^{n}$. Exactly as in the compact case, it can be shown that the sequence $\log N\left(\alpha^{n}\right)$ is subadditive, which implies the existence of the following limit

$$
h(T, \alpha)=\lim _{n \rightarrow \infty} \frac{1}{n} \log N\left(\alpha^{n}\right) .
$$

The topological entropy of the map $T$ is thus defined as

$$
h(T)=\sup _{\alpha} h(T, \alpha),
$$

where the supremum is taken over all admissible coverings $\alpha$ of $X$. We note that, when $X$ is already compact, the above definition coincides with the AKM definition, since thus every continuous map is proper and every open and finite covering of $X$ is admissible.

The following result generalizes, for non-compact spaces, the relation of the topological entropies of two semi-conjugated maps.

Proposition 2.1 Let $T: X \rightarrow X$ and $S: Y \rightarrow Y$ be two proper maps, where $X$ and $Y$ are topological spaces. If $f: Y \rightarrow X$ is a proper surjective map such that $f \circ S=T \circ f$, then we have that $h(T) \leq h(S)$.

Proof: Let $\alpha$ be an admissible covering of $X$. Since $f$ is a proper map, it follows that

$$
f^{-1}(\alpha)=\left\{f^{-1}(A): A \in \alpha\right\}
$$


is an admissible covering of $Y$. We claim that if $\beta$ is a subset of $\alpha^{n}$, then $f^{-1}(\beta)$ is a subset of $f^{-1}(\alpha)^{n}$. In fact, $B \in \beta$ if and only if

$$
B=A_{0} \cap T^{-1}\left(A_{1}\right) \cap \ldots \cap T^{-n}\left(A_{n}\right)
$$

where $A_{i} \in \alpha$, for each $i \in\{0, \ldots, n\}$. Thus we have that

$$
\begin{aligned}
f^{-1}(B) & =f^{-1}\left(A_{0}\right) \cap f^{-1}\left(T^{-1}\left(A_{1}\right)\right) \cap \ldots \cap f^{-1}\left(T^{-n}\left(A_{n}\right)\right) \\
& =f^{-1}\left(A_{0}\right) \cap S^{-1}\left(f^{-1}\left(A_{1}\right)\right) \cap \ldots \cap S^{-n}\left(f^{-1}\left(A_{n}\right)\right),
\end{aligned}
$$

where we used that $f^{-1} \circ T^{-i}=S^{-i} \circ T^{-1}$, since $f \circ S=T \circ f$. Thus it follows that $f^{-1}(\beta) \subset f^{-1}(\alpha)^{n}$. Reciprocally, we claim that if $\gamma$ is a subset of $f^{-1}(\alpha)^{n}$, then $\gamma=f^{-1}(\beta)$, where $\beta$ is some subset of $\alpha^{n}$. Proceeding analogously, if $C \in \gamma$, then

$$
\begin{aligned}
C & =f^{-1}\left(A_{0}\right) \cap S^{-1}\left(f^{-1}\left(A_{1}\right)\right) \cap \ldots \cap S^{-n}\left(f^{-1}\left(A_{n}\right)\right) \\
& =f^{-1}\left(A_{0} \cap T^{-1}\left(A_{1}\right) \cap \ldots \cap T^{-n}\left(A_{n}\right)\right),
\end{aligned}
$$

where $A_{i} \in \alpha$, for each $i \in\{0, \ldots, n\}$. Thus it follows that $C=f^{-1}(B)$, for some $B \in \alpha^{n}$, which implies that $\gamma=f^{-1}(\beta)$, where $\beta$ is some subset of $\alpha^{n}$.

If $\beta$ is a sub-covering of $\alpha^{n}$, then $f^{-1}(\beta)$ is a sub-covering of $f^{-1}(\alpha)^{n}$. Reciprocally, if $\gamma$ is a sub-covering of $f^{-1}(\alpha)^{n}$, then $\gamma=f^{-1}(\beta)$, where $\beta$ is some sub-covering of $\alpha^{n}$. In fact, we have already known that $\gamma=f^{-1}(\beta)$, for some subset $\beta$ of $\alpha^{n}$. We have to show just that $\beta$ is a covering of $X$. But this is immediate, since $\gamma=f^{-1}(\beta)$ is a covering of $Y, f$ is surjective and $B=f\left(f^{-1}(B)\right)$.

Hence we have that $N\left(\alpha^{n}\right)=N\left(f^{-1}(\alpha)^{n}\right)$ and taking logarithms, dividing by $n$ and taking limits, it follows that

$$
h(T, \alpha) \leq h\left(S, f^{-1}(\alpha)\right) \leq h(S)
$$

Since $\alpha$ is an arbitrary admissible covering of $X$, we get that $h(T) \leq h(S)$.

Now we introduce the concept of entropy associated to some given metric in two different ways. First we remember the classical definition, introduced in [2]. Given a metric space $(X, d)$ and a continuous map $T: X \rightarrow X$, we consider the metric given by

$$
d_{n}(x, y)=\max \left\{d\left(T^{i}(x), T^{i}(y)\right): 0 \leq i \leq n\right\}
$$


which is equivalent to the original metric $d$. Given a subset $Y \subset X$, a subset $G$ is an $(n, \varepsilon)$-generator of $Y$ if and only if, for every $y \in Y$, there exists $x \in G$ such that $d_{n}(x, y)<\varepsilon$. In other words, the collection of all $\varepsilon$-balls of $d_{n}$ centered at points of $G$ is in fact a covering of $Y$. We denote by $G_{n}(\varepsilon, Y)$ the smallest cardinality of all $(n, \varepsilon)$-generators of $Y$ and define

$$
g(\varepsilon, Y)=\lim _{n \rightarrow \infty} \sup \frac{1}{n} \log G_{n}(\varepsilon, Y) .
$$

It can be proved that $g(\varepsilon, Y)$ is monotone with respect to $\varepsilon$, so we can define

$$
h_{d}(T, Y)=\lim _{\varepsilon \downarrow 0} g(\varepsilon, Y) .
$$

The $d$-entropy of the map $T$ is thus defined as

$$
h_{d}(T)=\sup _{K} h_{d}(T, K),
$$

where the supremum is taken over all compact subsets $K$ of $X$.

The definition of $d$-entropy which we present in this paper is given by

$$
h^{d}(T)=\sup _{Y} h_{d}(T, Y),
$$

where now the supremum is taken over all subsets $Y$ of $X$ instead of just the compact ones. Note that here the metric $d$ lies at the superscript. Since we have that $h_{d}(T, Y)$ is monotone with respect to $Y$, it follows that

$$
h^{d}(T)=h_{d}(T, X) .
$$

We immediately notice that always $h_{d}(T) \leq h^{d}(T)$ and, when $X$ is itself compact, we get the equality. Denoting $G_{n}(\varepsilon, X)$ and $g(\varepsilon, X)$, respectively, by $G_{n}(\varepsilon)$ and $g(\varepsilon)$, we get that

$$
h^{d}(T)=\lim _{\varepsilon \downarrow 0} g(\varepsilon)
$$

and that

$$
g(\varepsilon)=\lim _{n \rightarrow \infty} \sup \frac{1}{n} \log G_{n}(\varepsilon) .
$$

Our first result is a generalization of the well know result which states that, if $(X, d)$ is compact, then the topological and the metric entropies coincide. In the non-compact case, this remains true but we need to ask for some regularity in the metric. Let $(X, d)$ be a metric space. The metric $d$ is admissible if the following conditions are verified: 
(1) If $\alpha_{\delta}=\left\{B\left(x_{1}, \delta\right), \ldots, B\left(x_{k}, \delta\right)\right\}$ is a covering of $X$, for every $\delta \in(a, b)$, where $0<a<b$, then there exists $\delta_{\varepsilon} \in(a, b)$ such that $\alpha_{\delta_{\varepsilon}}$ is admissible.

(2) Every admissible covering of $X$ has a Lebesgue number.

We observe that, if $(X, d)$ is compact, then $d$ is automatically admissible.

Proposition 2.2 Let $T: X \rightarrow X$ be a proper map, where $(X, d)$ is a metric space. If $d$ is an admissible metric, then $h(T)=h^{d}(T)$.

Proof: First we claim that, for any admissible covering $\alpha$ of $X$, it follows that $g(|\alpha|) \leq h(T, \alpha)$, where $|\alpha|$ is the maximum of the diameters of $A \in \alpha$. In fact, the elements of $\alpha^{n}$ are given by $A_{0} \cap T^{-1}\left(A_{1}\right) \cap \ldots \cap T^{-n}\left(A_{n}\right)$, where $A_{i} \in$ $\alpha$. Let $\beta$ be a sub-covering of $\alpha^{n}$. For each $B \in \beta$, take an $x \in B$ and consider $G$ the set of all such points. We claim that $G$ is an $(n,|\alpha|)$-generator set of $X$. In fact, let $y \in X$ and take some $A_{0} \cap T^{-1}\left(A_{1}\right) \cap \ldots \cap T^{-n}\left(A_{n}\right) \in \beta$ containing $y$. Taking $x \in G$ such that $x \in A_{0} \cap T^{-1}\left(A_{1}\right) \cap \ldots \cap T^{-n}\left(A_{n}\right)$, we have that $d\left(T^{i}(x), T^{i}(y)\right)<|\alpha|$, for every $i \in\{0,1, \ldots, n\}$, since $T^{i}(x), T^{i}(y) \in A_{i}$. Hence, for each sub-covering $\beta$ of $\alpha^{n}$, there exists an $(n,|\alpha|)$-generator set of $X$ such that $G_{n}(|\alpha|) \leq \# G \leq \# \beta$. Thus we get that $G_{n}(|\alpha|) \leq N\left(\alpha^{n}\right)$. Taking logarithms, dividing by $n$ and taking limits, it follows as claimed that $g(|\alpha|) \leq h(T, \alpha)$.

Now we claim that, for all $\varepsilon>0$, there exists $\delta_{\varepsilon} \in(\varepsilon / 2, \varepsilon)$ such that $h\left(T, \alpha_{\delta_{\varepsilon}}\right) \leq g(\varepsilon / 2)$, where $\alpha_{\delta_{\varepsilon}}$ is an admissible covering of balls with radius equals to $\delta_{\varepsilon}$. In fact, if $G=\left\{x_{1}, \ldots, x_{k}\right\}$ is an $(n, \varepsilon / 2)$-generator of $X$, for every $\delta \in(\varepsilon / 2, \varepsilon)$, we have that

$$
\beta_{\delta}=\left\{B\left(x_{i}, \delta\right) \cap \ldots \cap T^{-n}\left(B\left(T^{n}\left(x_{i}\right), \delta\right)\right): x_{i} \in G\right\}
$$

is a covering of $X$. To see this, given $x \in X$, there exists $x_{i} \in G$ such that $d_{n}\left(x, x_{i}\right)<\varepsilon / 2<\delta$ and thus we have that

$$
x \in B\left(x_{i}, \delta\right) \cap \ldots \cap T^{-n}\left(B\left(T^{n}\left(x_{i}\right), \delta\right)\right) .
$$

This implies that

$$
\alpha_{\delta}=\left\{B\left(T^{l}\left(x_{i}\right), \delta\right): x_{i} \in G, 0 \leq l \leq n\right\}
$$

is a finite covering of $X$, for every $\delta \in(\varepsilon / 2, \varepsilon)$. Since $d$ is an admissible metric, there exists $\delta_{\varepsilon} \in(\varepsilon / 2, \varepsilon)$ such that $\alpha_{\delta_{\varepsilon}}$ is an admissible covering of 
balls with radius equals to $\delta_{\varepsilon}$. Furthermore we have that $\beta_{\delta_{\varepsilon}}$ is a sub-covering of $\alpha_{\delta_{\varepsilon}}^{n}$. Hence, for each $(n, \varepsilon / 2)$-generator $G$ of $X$, there exist $\delta_{\varepsilon} \in(\varepsilon / 2, \varepsilon)$ and a sub-covering $\beta_{\delta_{\varepsilon}}$ of $\alpha_{\delta_{\varepsilon}}^{n}$ such that $N\left(\alpha_{\delta_{\varepsilon}}\right) \leq \# \beta_{\delta_{\varepsilon}} \leq \# G$. Thus it follows that $N\left(\alpha_{\delta_{\varepsilon}}\right) \leq G_{n}(\varepsilon / 2)$. Taking logarithms, dividing by $n$ and taking limits, we get as claimed that there exists $\delta_{\varepsilon} \in(\varepsilon / 2, \varepsilon)$ such that $h\left(T, \alpha_{\delta_{\varepsilon}}\right) \leq g(\varepsilon / 2)$. Since $\left|\alpha_{\delta_{\varepsilon}}\right| \leq 2 \delta_{\varepsilon} \leq 2 \varepsilon$, we have that

$$
g(2 \varepsilon) \leq g\left(\left|\alpha_{\delta_{\varepsilon}}\right|\right) \leq h\left(T, \alpha_{\delta_{\varepsilon}}\right) \leq g(\varepsilon / 2) .
$$

Taking limits with $\varepsilon \downarrow 0$, it follows that

$$
h^{d}(T)=\lim _{\varepsilon \downarrow 0} h\left(T, \alpha_{\delta_{\varepsilon}}\right)=\sup _{\varepsilon>0} h\left(T, \alpha_{\delta_{\varepsilon}}\right) .
$$

In order to complete the proof, it remains to show that the above supremum is equal to $h(T)$. For any admissible covering $\alpha$ of $X$, take $\varepsilon$ a Lebesgue number of this covering. We claim that $N\left(\alpha^{n}\right) \leq N\left(\alpha_{\delta_{\varepsilon}}^{n}\right)$, where $\alpha_{\delta_{\varepsilon}}$ is given above. In fact, since every element of $\alpha_{\delta_{\varepsilon}}^{n}$ if given by $B_{0} \cap \ldots \cap T^{-n}\left(B_{n}\right)$, where $\left\{B_{0}, \ldots, B_{n}\right\}$ are balls of radius $\delta_{\varepsilon}<\varepsilon$, there exist $\left\{A_{0}, \ldots, A_{n}\right\} \subset \alpha$ such that $B_{i} \subset A_{i}$, for all $i \in\{0, \ldots, n\}$. Thus we have that

$$
B_{0} \cap \ldots \cap T^{-n}\left(B_{n}\right) \subset A_{0} \cap \ldots \cap T^{-n}\left(A_{n}\right),
$$

showing that, for each sub-covering $\beta$ of $\alpha_{\delta_{\varepsilon}}^{n}$, there exists a sub-covering $\gamma$ of $\alpha^{n}$ such that $N\left(\alpha^{n}\right) \leq \# \gamma \leq \# \beta$. Hence we get as claimed that $N\left(\alpha^{n}\right) \leq N\left(\alpha_{\delta_{\varepsilon}}^{n}\right)$. Taking logarithms, dividing by $n$ and taking limits, it follows that

$$
h(T, \alpha) \leq h\left(T, \alpha_{\delta_{\varepsilon}}\right) \leq \sup _{\varepsilon>0} h\left(T, \alpha_{\delta_{\varepsilon}}\right)
$$

which shows that

$$
h(T)=\sup _{\varepsilon>0} h\left(T, \alpha_{\delta_{\varepsilon}}\right)
$$

completing the proof.

From now one we assume that $X$ is a locally compact space. Thus we have associated to $X$ its one point compactification, which we will denote by $\widetilde{X}$. We have that $\widetilde{X}$ is defined as the disjoint union of $X$ with $\{\infty\}$, where $\underset{\widetilde{X}}{\infty}$ is some point not in $X$ called the point at the infinity. The topology in $\widetilde{X}$ consists by the former open sets in $X$ and by the sets $A \cup\{\infty\}$, where 
the the complement of $A$ in $X$ is compact. If $T: X \rightarrow X$ is a proper map, defining $\widetilde{T}: \widetilde{X} \rightarrow \widetilde{X}$ by

$$
\widetilde{T}(\widetilde{x})=\left\{\begin{array}{ll}
T(\widetilde{x}), & x \neq \infty \\
\infty, & x=\infty
\end{array},\right.
$$

we have that $\widetilde{T}$ is also proper map, called the extension of $T$ to $\widetilde{X}$. To see this, we only need to verify that $\widetilde{T}$ is continuous at $\infty$. If $A \cup\{\infty\}$ is a neighborhood of $\infty$, then the complement of $A$ is compact and we have that

$$
\widetilde{T}^{-1}(A \cup\{\infty\})=T^{-1}(A) \cup\{\infty\}
$$

is also a neighborhood of $\infty$, since $T$ is proper and thus the complement of $T^{-1}(A)$ is also compact.

The following result shows that the restriction to $X$ of any metric on $\tilde{X}$ is always admissible and that their respective entropies coincide.

Proposition 2.3 Let $T: X \rightarrow X$ be a proper map, where $X$ is a locally compact separable space. Let $d$ be the metric given by the restriction to $X$ of some metric $\widetilde{d}$ on $\widetilde{X}$, the one point compactification of $X$. Then it follows that $d$ is an admissible metric and that

$$
h^{d}(T)=h^{\widetilde{d}}(\widetilde{T})
$$

where $\widetilde{T}$ is the extension of $T$ to $\widetilde{X}$. In particular, we get that $h(T)=h(\widetilde{T})$.

Proof: First we show that $G_{n}(\varepsilon)$ is finite for every $\varepsilon>0$. Let $\widetilde{G}=$ $\left\{\widetilde{x}_{1}, \ldots, \widetilde{x}_{k}\right\} \subset \widetilde{X}$ be an $(n, \varepsilon / 2)$-generator set of $\widetilde{X}$. By the density of $X$ in $\widetilde{X}$, it follows that there exist $\left\{x_{1}, \ldots, x_{k}\right\} \subset X$, such that $\widetilde{d}_{n}\left(x_{i}, \widetilde{x}_{i}\right)<\varepsilon / 2$. If $x \in X \subset \widetilde{X}$, we have that $\widetilde{d}_{n}\left(x, \widetilde{x}_{i}\right)<\varepsilon / 2$, for some $\widetilde{x}_{i} \in \widetilde{G}$. Hence it follows that

$$
d_{n}\left(x, x_{i}\right) \leq \widetilde{d}_{n}\left(x, \widetilde{x}_{i}\right)+\widetilde{d}_{n}\left(x_{i}, \widetilde{x}_{i}\right)<\varepsilon / 2+\varepsilon / 2=\varepsilon,
$$

showing that $G=\left\{x_{1}, \ldots, x_{k}\right\}$ is an $(n, \varepsilon)$-generator set of $X$. If we choose $\widetilde{G}$ such that $\# \widetilde{G}=\widetilde{G}_{n}(\varepsilon / 2)$, then we get as claimed that

$$
G_{n}(\varepsilon) \leq \widetilde{G}_{n}(\varepsilon / 2)<\infty .
$$


Now assume that $\alpha_{\delta}=\left\{B\left(x_{1}, \delta\right), \ldots, B\left(x_{k}, \delta\right)\right\}$ is a covering of $X$, for every $\delta \in(a, b)$, where $0<a<b$. Since, for each fixed $\delta$, the number of balls are finite, it follows that there exists $\delta_{\varepsilon} \in(a, b)$ such that $\infty \notin \widetilde{S}\left(x_{i}, \delta_{\varepsilon}\right)$, for every $i \in\{1, \ldots, k\}$, where $\widetilde{S}(x, r)$ is the sphere in $\widetilde{X}$ of radius $r$ centered in $x$. Denoting by $\widetilde{B}(x, r)$ the open ball in $\widetilde{X}$ of radius $r$ centered in $x$, it remains two alternatives: 1$)$ the point $\infty$ is inside $\widetilde{B}\left(x_{i}, \delta_{\varepsilon}\right)$ or 2$)$ the point $\infty$ is not in the closure of $\widetilde{B}\left(x_{i}, \delta_{\varepsilon}\right)$. In the first case, the complement of $B\left(x_{i}, \delta_{\varepsilon}\right)$ in $X$ is equal to the complement of $\widetilde{B}\left(x_{i}, \delta_{\varepsilon}\right)$ in $X$, which is compact. In the second alternative, there exist a open neighborhood $U$ of $\infty$ which has empty intersection with $\widetilde{B}\left(x_{i}, \delta_{\varepsilon}\right)$. Thus $B\left(x_{i}, \delta_{\varepsilon}\right)=\widetilde{B}\left(x_{i}, \delta_{\varepsilon}\right)$ is in the complement of $U$ in $X$, which is compact. This shows that the closure or the complement of $B\left(x_{i}, \delta_{\varepsilon}\right)$ in $X$ is compact, showing that $\alpha_{\delta_{\varepsilon}}$ is already admissible.

We show now that every admissible covering of $X$ has a Lebesgue number. If $\alpha$ is admissible covering of $X$, there exists an open covering $\widetilde{\alpha}$ of $\widetilde{X}$ such that $A \in \alpha$ if and only if there exists $\widetilde{A} \in \widetilde{\alpha}$, with $A=\widetilde{A} \cap X$. In fact, if $\alpha$ is an admissible covering of $X$, there exists at least one $A \in \alpha$ with compact complement in $X$ and such that its closure in $X$ is not compact. If we define $\widetilde{A}=A \cup\{\infty\}$, we have that $\widetilde{A}$ is an open neighborhood of $\{\infty\}$ in $\widetilde{X}$. Thus defining

$$
\widetilde{\alpha}=\{\widetilde{A}\} \cup\{B \in \alpha: B \neq A\},
$$

it follows as claimed that $\widetilde{\alpha}$ is an open covering of $\widetilde{X}$ such that $A \in \alpha$ if and only if there exists $\widetilde{A} \in \widetilde{\alpha}$, with $A=\widetilde{A} \cap X$. If $\varepsilon$ is a Lebesgue number for $\widetilde{\alpha}$, then we claim that $\varepsilon$ is a also a Lebesgue number for $\alpha$. To see this, if $B(x, \varepsilon)$ is a ball in $X$, there exists $\widetilde{A} \in \widetilde{\alpha}$ such that $B(x, \varepsilon) \subset \widetilde{A}$. Thus it follows that $B(x, \varepsilon) \subset A$, where $A=\widetilde{A} \cap X \in \alpha$, completing the proof that $d$ is an admissible metric.

Finally we show that $h^{d}(T)=h^{\widetilde{d}}(\widetilde{T})$. Let $G=\left\{x_{1}, \ldots, x_{k}\right\}$ be an $(n, \varepsilon / 2)$-generator set of $X$. By the density of $X$ in $\widetilde{X}$, if $\widetilde{x} \in \widetilde{X}$, there exists $x \in X$ such that $\widetilde{d}_{n}(\widetilde{x}, x)<\varepsilon / 2$, since $d_{n}$ is topologically equivalent to $d$. Thus we have that $d_{n}\left(x, x_{i}\right)<\varepsilon / 2$, for some $x_{i} \in G$. Hence it follows that

$$
\widetilde{d}_{n}\left(\widetilde{x}, x_{i}\right) \leq \widetilde{d}_{n}(\widetilde{x}, x)+d_{n}\left(x, x_{i}\right)<\varepsilon / 2+\varepsilon / 2=\varepsilon,
$$

showing that $G=\left\{x_{1}, \ldots, x_{k}\right\}$ is an $(n, \varepsilon)$-generator set of $\widetilde{X}$. Choosing $G$ such that $\# G=G_{n}(\varepsilon / 2)$, we get that $\widetilde{G}_{n}(\varepsilon) \leq G_{n}(\varepsilon / 2)$. Since we have also shown above that $G_{n}(\varepsilon) \leq \widetilde{G}_{n}(\varepsilon / 2)$, taking logarithms, dividing by $n$ and 
taking limits, we get that

$$
g(\varepsilon) \leq \widetilde{g}(\varepsilon / 2) \quad \text { and } \quad \widetilde{g}(\varepsilon) \leq g(\varepsilon / 2) .
$$

Therefore it follows that

$$
h^{d}(T)=\lim _{\varepsilon \downarrow 0} g(4 \varepsilon) \leq \lim _{\varepsilon \downarrow 0} \widetilde{g}(2 \varepsilon)=h^{\widetilde{d}}(\widetilde{T}) \leq \lim _{\varepsilon \downarrow 0} g(\varepsilon)=h^{d}(T),
$$

showing that $h^{d}(T)=h^{\widetilde{d}}(\widetilde{T})$. The last statement now follows applying Proposition 2.2.

\section{The variational principle}

In this section, we present a full extension for non-compact sets of the well known variational principle involving entropies. We start with a proper map $T: X \rightarrow X$ and remember the concept of entropy associated to some given $T$-invariant probability on $X$. A T-invariant probability on $X$ is a Borel measure $\mu$ such that $\mu(X)=1$ and $\mu\left(T^{-1}(A)\right)=\mu(A)$, for all Borel subsets $A \subset X$. We denote by $\mathcal{P}_{T}(X)$ the set of all $T$-invariant probability on $X$. A Borel partition $\mathcal{A}$ of $X$ is a partition of $X$ where all of its elements are Borel sets. Given a finite Borel partition $\mathcal{A}$ of $X$, for every $n \in \mathbb{N}$, we have that the set given by

$$
\mathcal{A}^{n}=\left\{A_{0} \cap T^{-1}\left(A_{1}\right) \cap \ldots \cap T^{-n}\left(A_{n}\right): A_{i} \in \mathcal{A}\right\}
$$

is also a finite Borel partition of $X$, since $T$ is continuous. For a given finite Borel partition $\mathcal{A}$ of $X$, we define its associated $n$-entropy as

$$
H\left(\mathcal{A}^{n}\right)=\sum_{B \in \mathcal{A}^{n}} \phi(\mu(B))
$$

where $\phi:[0,1] \rightarrow \mathbb{R}$ is the continuous function given by

$$
\phi(x)=\left\{\begin{array}{cl}
-x \log (x), & x \in(0,1] \\
0, & x=0
\end{array} .\right.
$$

It can be shown that the sequence $H\left(\mathcal{A}^{n}\right)$ is subadditive, which implies the existence of the following limit

$$
h(T, \mathcal{A})=\lim _{n \rightarrow \infty} \frac{1}{n} H\left(\mathcal{A}^{n}\right) .
$$


The $\mu$-entropy of the map $T$ is thus defined as

$$
h_{\mu}(T)=\sup _{\mathcal{A}} h(T, \mathcal{A})
$$

where the supremum is taken over all finite Borel partition $\mathcal{A}$ of $X$. The next result was first proved in the Lemma 1.5 of [4], where they used the fact that the supremum of the measure entropies taken over all invariant probabilities is equals to that one just taken over all ergodic invariant probabilities. We present here an elementary proof of the quoted result which dos not use this fact. In the following $\mathcal{P}_{T}(X)$ denotes the set of all $T$-invariant probabilities on $X$.

Lemma 3.1 Let $T: X \rightarrow X$ be a proper map and $\widetilde{T}: \widetilde{X} \rightarrow \widetilde{X}$ be its extension in the one point compactification $\widetilde{X}$ of the locally compact separable space $X$. Then it follows that

$$
\sup _{\mu} h_{\mu}(T)=\sup _{\widetilde{\mu}} h_{\widetilde{\mu}}(\widetilde{T})
$$

where the suprema are taken, respectively, over $\mathcal{P}_{T}(X)$ and over $\mathcal{P}_{\widetilde{T}}(\widetilde{X})$.

Proof: If $\mu \in \mathcal{P}_{T}(X)$, definig $\widetilde{\mu}(\widetilde{A})=\mu(\widetilde{A} \cap X)$, it is immediate that $\widetilde{\mu} \in \mathcal{P}_{\widetilde{T}}(\widetilde{X})$, since $X$ and $\{\infty\}$ are $\widetilde{T}$-invariant sets. It is also immediate that $h_{\mu}(T)=h_{\widetilde{\mu}}(\widetilde{T})$, showing that

$$
\sup _{\mu} h_{\mu}(T) \leq \sup _{\widetilde{\mu}} h_{\widetilde{\mu}}(\widetilde{T})
$$

Now let $\widetilde{\mu} \in \mathcal{P}_{\widetilde{T}}(\widetilde{X})$. If $\widetilde{\mu}(\infty)=1$, it is immediate that $h_{\widetilde{\mu}}(\widetilde{T})=0$, since $X$ and $\{\infty\}$ are $\widetilde{T}$-invariant sets. Thus we can assume that $\widetilde{\mu}(\infty)=c<1$. It is also immediate that

$$
\mu=\left.\left(\frac{1}{1-c}\right) \widetilde{\mu}\right|_{\mathcal{B}(X)} \in \mathcal{P}_{T}(X) .
$$

We claim that $h_{\widetilde{\mu}}(\widetilde{T}) \leq h_{\mu}(T)$. If $\widetilde{\mathcal{A}}=\left\{\widetilde{A}_{1}, \ldots, \widetilde{A}_{l}\right\}$ is a measurable partition of $\tilde{X}$, defining $A_{i}=\widetilde{A}_{i} \cap X$, it follows that $\mathcal{A}=\left\{A_{1}, \ldots, A_{l}\right\}$ is a 
measurable partition of $X$. We have that, $B \in \mathcal{A}^{n}$ if and only if there exists $\widetilde{B} \in \widetilde{\mathcal{A}}^{n}$, with $B=\widetilde{B} \cap X$. This follows, because

$$
\widetilde{T}^{-j}\left(\widetilde{A}_{i}\right) \cap X=T^{-j}\left(A_{i}\right)
$$

for any $j \in\{0, \ldots, n\}$ and any $i \in\{1, \ldots, l\}$, since $X$ and $\{\infty\}$ are $\widetilde{T}$ invariant sets. It follows that

$$
H\left(\widetilde{\mathcal{A}}^{n}\right)=\sum_{\widetilde{B} \in \widetilde{\mathcal{A}}^{n}} \phi(\widetilde{\mu}(\widetilde{B}))=\phi\left(\widetilde{\mu}\left(\widetilde{B}_{\infty}\right)\right)+\sum_{\widetilde{B} \neq \widetilde{B}_{\infty}} \phi(\widetilde{\mu}(\widetilde{B})),
$$

where $\infty \in \widetilde{B}_{\infty}$. Since $\widetilde{\mu}(\widetilde{B})=(1-c) \mu(B)$, for each $\widetilde{B} \neq \widetilde{B}_{\infty}$, it follows that

$$
H\left(\widetilde{\mathcal{A}}^{n}\right)=b+\sum_{B \in \mathcal{A}^{n}} \phi(a \mu(B)),
$$

where $a=1-c$ and $b=\phi\left(\widetilde{\mu}\left(\widetilde{B}_{\infty}\right)\right)-\phi(a \mu(B))$. Hence

$$
\begin{aligned}
H\left(\widetilde{\mathcal{A}}^{n}\right) & =b-\sum_{B \in \mathcal{A}^{n}} a \mu(B) \log (a \mu(B)) \\
& =b-a\left(\sum_{B \in \mathcal{A}^{n}} \mu(B)(\log (a)+\log (\mu(B)))\right. \\
& =b-a \log (a)\left(\sum_{B \in \mathcal{A}^{n}} \mu(B)\right)+a\left(\sum_{B \in \mathcal{A}^{n}} \phi(\mu(B))\right) \\
& =b+\phi(a)+a H\left(\mathcal{A}^{n}\right) .
\end{aligned}
$$

It follows that $H\left(\widetilde{\mathcal{A}}^{n}\right) \leq d+H\left(\mathcal{A}^{n}\right)$, since $a=1-c \leq 1$ and

$$
b+\phi(a) \leq d=2 \max \{\phi(x): x \in[0,1]\} .
$$

Dividing by $n$ and taking limits, we get that

$$
h_{\widetilde{\mu}}(\widetilde{T}, \widetilde{\mathcal{A}}) \leq h_{\mu}(T, \mathcal{A}) \leq h_{\mu}(T) .
$$

Since $\widetilde{\mathcal{A}}$ is arbitrary, we have that

$$
h_{\widetilde{\mu}}(\widetilde{T}) \leq h_{\mu}(T) \leq \sup _{\mu} h_{\mu}(T) .
$$


Since $\widetilde{\mu} \in \mathcal{P}_{\widetilde{T}}(\widetilde{X})$ is also arbitrary, it follows that

$$
\sup _{\widetilde{\mu}} h_{\widetilde{\mu}}(\widetilde{T}) \leq \sup _{\mu} h_{\mu}(T)
$$

completing the proof.

Now we prove the variational principle for entropies in a locally compact separable space. In Lemma 1.5 of [4], it was proved that

$$
\sup _{\mu} h_{\mu}(T)=\inf _{d} h_{d}(T) .
$$

Here we improve this result showing that the above infimum is always attained at $d$, whenever $d$ is an admissible metric. Moreover the supremum and the minimum coincide with the topological entropy introduced in the Section 2 ,

Theorem 3.2 Let $T: X \rightarrow X$ be a continuous map, where $X$ is a locally compact separable space. Then it follows that

$$
\sup _{\mu} h_{\mu}(T)=h(T)=\min _{d} h_{d}(T),
$$

where the minimum is attained whenever $d$ is an admissible metric.

Proof: The the variational principle for compact spaces states that

$$
h(\widetilde{T})=\sup _{\widetilde{\mu}} h_{\widetilde{\mu}}(\widetilde{T}) .
$$

By Proposition 1.4 of [4], we have that

$$
\sup _{\mu} h_{\mu}(T) \leq \inf _{d} h_{d}(T) .
$$

Applying Lemma 3.1, it follows that

$$
h(\widetilde{T})=\sup _{\mu} h_{\mu}(T) \leq \inf _{d} h_{d}(T) .
$$

Applying Propositions 2.2 and 2.3, we get that

$$
h_{d}(T) \leq h^{d}(T)=h(T)=\sup _{\mu} h_{\mu}(T) \leq \inf _{d} h_{d}(T),
$$

where, in the first two terms, $d$ is any admissible metric. 


\section{Topological entropy of automorphisms}

In this section, we compute the topological entropy for automorphisms of simply connected nilpotent Lie groups. We start with linear isomorphisms

of a finite dimensional vector space. For this, we need to determine the recurrent set of a linear isomorphism in terms of its multiplicative Jordan decomposition (see Lema 7.1, page 430, of [5]). If $T: V \rightarrow V$ is a linear isomorphism, where $V$ is a finite dimensional vector space, then we can write $T=T_{H} T_{E} T_{U}$, where $T_{H}: V \rightarrow V$ is diagonalizable in $V$ with positive eigenvalues, $T_{E}: V \rightarrow V$ is an isometry relative to some appropriate norm and $T_{U}: V \rightarrow V$ is a linear isomorphism which can be decomposed into the sum of the identity map plus some nilpotent linear map. The linear maps $T_{H}$, $T_{E}$ and $T_{U}$ commute, are unique and called, respectively, the hyperbolic, the elliptic and the unipotent components of the multiplicative Jordan decomposition of $T$. In the next result, we prove that the recurrent set $R(T)$ of $T$ is given as the intersection of the fixed points of the hyperbolic and unipotent components. Using this characterization, we also show that the topological entropy of $T$ always vanishes.

Proposition 4.1 Let $T: V \rightarrow V$ be a linear isomorphism, where $V$ is a finite dimensional vector space. Then the recurrent set of $T$ is given by

$$
R(T)=\operatorname{fix}\left(T_{H}\right) \cap \operatorname{fix}\left(T_{U}\right) .
$$

Furthermore, it follows that $h(T)=0$.

Proof: Let $\mathbb{P} T: \mathbb{P} V \rightarrow \mathbb{P} V$ be the map induced by $T$ in the projective space of $V$ and, for a subspace $W \subset V$, denote by $\mathbb{P} W$ its projection in $\mathbb{P} V$. By Proposition 2 of [3], we have that

$$
R(\mathbb{P} T)=\operatorname{fix}\left(\mathbb{P} T_{H}\right) \cap \text { fix }\left(\mathbb{P} T_{U}\right) .
$$

By linearity, it is immediate that $\mathbb{P}\langle R(T)\rangle \subset R(\mathbb{P} T)$, where $\langle A\rangle$ denotes the linear subspace generated by $A \subset V$. Thus it follows that

$$
R(T) \subset \operatorname{eig}\left(T_{H}\right) \cap \operatorname{eig}\left(T_{U}\right)
$$

where eig $(S)$ denotes the union of the eigenspaces of a linear map $S: V \rightarrow V$. Since $T_{U}$ is unipotent, all of its eigenvalues are equals to one, implying that $\operatorname{eig}\left(T_{U}\right)=\operatorname{fix}\left(T_{U}\right)$. Hence $R(T) \subset \operatorname{eig}\left(T_{H}\right) \cap \operatorname{fix}\left(T_{U}\right)$. Now let $v \in R(T)$ 
be such that $T_{H} v=\lambda v$, for some $\lambda>0$. Since the multiplicative Jordan decomposition is commutative, it follows that

$$
\left|T^{n} v\right|=\left|T_{E}^{n} T_{H}^{n} T_{U}^{n} v\right|=\left|T_{H}^{n} v\right|=\lambda^{n}|v|,
$$

where we used the fact that $T_{E}$ is an isometry relative to some appropriate norm $|\cdot|$. This shows that $\lambda$ is equals to one and thus that $R(T)$ is contained in fix $\left(T_{H}\right) \cap$ fix $\left(T_{U}\right)$. Since fix $\left(T_{H}\right) \cap$ fix $\left(T_{U}\right)$ is invariant by $T_{E}$, we can consider the restriction of $T$ to this set, which is just the restriction of $T_{E}$. Thus we get that $R(T)=\operatorname{fix}\left(T_{H}\right) \cap \operatorname{fix}\left(T_{U}\right)$, since the restriction of $T_{E}$ to $\operatorname{fix}\left(T_{H}\right) \cap \operatorname{fix}\left(T_{U}\right)$ is an isometry whose orbits have compact closure.

Now to show that $h(T)=0$, we first note that, by the Poincaré recurrence theorem, for every $\mu \in \mathcal{P}_{T}(X)$, we have that $h_{\mu}(T)=h_{\mu}\left(\left.T\right|_{R(T)}\right)$, since $R(T)$ is a closed set. By the variational principle, it follows that

$$
h(T)=h\left(\left.T\right|_{R(T)}\right) \leq h_{d}\left(\left.T\right|_{R(T)}\right),
$$

for every metric $d$. Since $\left.T\right|_{R(T)}=\left.T_{E}\right|_{R(T)}$ is an isometry relative to some appropriate metric $d$, we get that $h_{d}\left(\left.T\right|_{R(T)}\right)=0$, completing the proof.

We note that the classical formula for the $d$-entropy of a linear isomorphism, where $d$ is the euclidian metric, is just an upper bound for its null topological entropy. Thus it might be an interesting problem to calculate the topological entropy of a given automorphism $\phi$ of a noncompact Lie group $G$, since the classical formula for its $d$-entropy, where $d$ is some invariant metric, is as well just an upper bound for its topological entropy. Remember that the classical formula is given by

$$
h_{d}(\phi)=\sum_{|\lambda|>1} \log |\lambda|,
$$

where $\lambda$ runs through the eigenvalues of $\mathrm{d}_{1} \phi: \mathfrak{g} \rightarrow \mathfrak{g}$, the differential of the automorphism $\phi$ at the identity element of $G$.

The following theorem gives an answer for the above problem when $G$ is a simply connected nilpotent Lie group.

Theorem 4.2 Let $\phi: G \rightarrow G$ be an automorphism, where $G$ is a simply connected nilpotent Lie group. Then it follows that $h(\phi)=0$. 
Proof: If $G$ is a connected and simply connected nilpotent Lie group, we have that the exponential map is a diffeomorphism between $\mathfrak{g}$ and $G$ (see Theorem 1.127, page 107, of [6]). Since $\phi(\exp (X))=\exp \left(\mathrm{d}_{1} \phi X\right)$, we get that $\phi$ and $\mathrm{d}_{1} \phi$ are conjugated maps. By Proposition 2.1, it follows that $h(\phi)=h\left(\mathrm{~d}_{1} \phi\right)=0$.

We note that, if the fundamental group of $G$ is not trivial, it is possible the existence of an automorphism with positive topological entropy, even when $G$ is abelian. In fact, the map $\phi(z)=z^{2}$ is an automorphism of the abelian Lie group $S^{1}$ and it is well known that it has topological entropy $h(\phi)=\log (2)>0$. We have that the canonical homomorphism $\pi: \mathbb{R} \rightarrow S^{1}$, given by $\pi(x)=e^{i x}$, is a semi-conjugation between $\phi$ and the automorphism $\widetilde{\phi}$ of the universal covering $\mathbb{R}$ of $S^{1}$, given by $\widetilde{\phi}(x)=2 x$. Although $h(\widetilde{\phi})=$ 0 , we can not apply Proposition 2.1 to conclude that $h(\phi)=0$, since the canonical homomorphism $\pi$ is a proper map if and only if the fundamental group of $G$ is finite.

\section{References}

[1] R. Adler, A. Konheim and H. MacAndrew : Topological entropy. Trans. Americ. Math Soc. 114 (1965), 309-319.

[2] R. Bowen : Topological entropy for noncompact sets. Trans. Americ. Math Soc. 184 (1973), 125-136.

[3] T. Ferraiol, M. Patrão, L. Santos and L. Seco : Multiplicative Jordan decomposition and dynamics on flag manifolds. preprint (2008).

[4] M. Handel and B. Kitchens: Metrics and entropy for non-compact spaces. Isr. J. Math., 91 (1995), 253-271.

[5] Helgason, S. Differential Geometry, Lie Groups and Symmetric Spaces. Academic Press, New York, (1978).

[6] Knapp, A. W. : Lie Groups Beyond an Introduction, Progress in Mathematics, v. 140, Birkhäuser, Boston (2004).

[7] P. Walters : An introduction to ergodic theory. GTM, Springer-Verlag, Berlin, 1981. 